\title{
L-theanine and Neumentix mixture improves sleep quality and modulates brain neurotransmitter levels in mice
}

\author{
Ying Zhang ${ }^{1 \#}$, Xiuzhen Jia ${ }^{2 \#}$, Ximin Chen ${ }^{3}$, Yajuan Liu ${ }^{1}$, Zifu Zhao ${ }^{4}$, Jingyu Hao ${ }^{4}$, Rui Wu ${ }^{5}$, Haotian Feng ${ }^{2}$, \\ Xiangnan $\operatorname{Ren}^{6}$ \\ ${ }^{1}$ Department of Nutrition and Food Hygiene, School of Public Health, Jilin University, Changchun, China; ${ }^{2}$ Yili Innovation Center, Inner Mongolia \\ Yili Industrial Group Co., Ltd., Hohhot, China; ${ }^{3}$ Bonnysci International Science \& Technology Co., Ltd., Beijing, China; ${ }^{4}$ Inner Mongolia Dairy \\ Technology Research Institute Co., Ltd., Hohhot, China; ${ }^{5}$ Department of Nutrition, Food Safety and Toxicology, West China School of Public \\ Health, Healthy Food Evaluation Research Center, Food Safety Monitoring and Risk Assessment Key Laboratory of Sichuan Province, Sichuan \\ University, Chengdu, China; ${ }^{6}$ Department of Food Nutrition \& Function Assessment Center, Beijing Institute of Nutritional Resources, Beijing, \\ China \\ Contributions: (I) Concept and design: X Chen, X Jia; (II) Administrative support: X Jia, Z Zhao, J Hao; (III) Provision of study materials for patients: \\ H Feng, X Ren, Y Liu; (IV) Data collection and assembly: X Chen, R Wu; (V) Data analysis and interpretation: X Chen, Y Zhang; (VI) Manuscript \\ writing: All authors; (VII) Final approval of manuscript: All authors. \\ \#These authors contributed equally to this work. \\ Correspondence to: Haotian Feng. Yili Innovation Center, Inner Mongolia Yili Industrial Group Co., Ltd., Hohhot 010110, China. \\ Email: fenghaotian@yili.com; Xiangnan Ren. Beijing Institute of Nutritional Sources, Beijing 100069, China. Email: renxiangnan2018@126.com.
}

Backgroundk L-theanine (L-THE), a natural amino acid found in green tea, has been shown to improve anxiety and sleep. Neumentix proprietary spearmint extract (PSE), which is commonly found in beverage flavoring a pharmaceutical, also has a wide range of health benefits, including cognitive performance improvement.

Methods: Four experiments tested the effects of L-THE and PSE on sleep: a direct sleeping test, pentobarbital-induced sleeping test, sub-hypnotic pentobarbital-induced sleeping test, and sodium barbitalinduced sleeping test. Presence of neurotransmitters in brain tissue was detected by liquid chromatography mass spectroscopy (HP LC-MS) during these studies.

Results: Pentobarbital-induced sleeping and sodium barbital-induced sleeping tests examined the potential effect of L-THE/PSE mixture on synergistic sleep, while neurotransmitter levels in the brain were determined by the high performance liquid chromatography/mass spectroscopy (HPLC/MS) method. L-THE and L-THE/PSE mixture showed increased sleep duration and shortened sleep latency when coadministrated with pentobarbital or sodium barbital. The mixture also increased sleeping rate when coadministrated with the pentobarbital at sub-hypnotic dose. Additionally, the L-THE, PSE and L-THE/PSE mixture significantly increased the concentrations of acetylcholine (Ach), $\gamma$-aminobutyric acid (GABA), and decreased the concentration of serotonin (5-HT) in the brain.

Conclusions: These data demonstrated that L-THE/PSE mixture regulates sleep disorders via the GABA receptor and neurotransmitter systems.

Keywords: L-theanine (L-THE); Neumentix; sleep improvement; neurotransmitters

Submitted Jan 31, 2021. Accepted for publication Apr 24, 2021.

doi: 10.21037/apm-21-663

View this article at: http://dx.doi.org/10.21037/apm-21-663 


\section{Introduction}

In recent years, insomnia has been shown to have serious physical and mental health effects. Sleep deprivation increases risk of various chronic health conditions, including obesity, diabetes, heart disease and mental health issues (1). While $25 \%$ to $35 \%$ of the population have suffered from occasional sleeplessness, 9 to $15 \%$ of people worldwide report chronic and severe insomnia (2-4).

In order to improve sleep quality, most insomniacs are known to take hypnotics, such as benzodiazepine analogues, zolpidem, and doxepin; however, these agents have several adverse effects, including drug dependency, memory loss, gastrointestinal issues, and daytime somnolence. Longterm use of these drugs can also cause muscle relaxation, addiction, and cognitive impairment (5). Therefore, exploration of new plant-derived biologically-active alternatives for sleep improvement, which may have fewer adverse effects, is paramount.

L-theanine ( $\gamma$-glutamylethylamide, L-THE), a nonprotein amino acid abundantly present in tea leaves, has a similar structure to glutamate and glutamine $(6,7)$. L-THE gets absorbed through microvilli via intestinal epithelial cells from where, it is transported to the brain tissues as it crosses the blood brain barrier. A number of studies have reported a variety of pharmacological effects of L-THE, including improved cognitive function (8), neuroprotection (9), improved anti-oxidative activity (10) and reduced psychological stress (11). Additionally, several studies have shown that L-THE can reduce sleep disturbances (12-14).

Neumentix [a spearmint-like extract, proprietary spearmint extract (PSE)] is a dietary supplement containing abundant rosmarinic acid and polyphenols (15). Some reports indicate that PSE has beneficial effects on learning and memory (16), anti-oxidative effects (17), antiinflammatory effects and attention enhancement (18). Rosmarinic and salvianolic acids found in PSE have also been shown to have anti-oxidative, anti-cancer, antibacterial, anti-inflammatory, neuroprotective and various other biological effects $(19,20)$; however, little research exists pertaining to its effect on sleep.

Previous studies have reported that L-THE and rosmarinic acid improve sleep behaviors (21); however, no studies report the effect of their mixtures on sleep. The present study aims to investigate the effects of L-THE and PSE on sleep improvement, and whether their combined use is more effective than use of the supplement alone. Furthermore, while monoamines in the brain play a key role in the sleep-wake cycle-such as $\gamma$-aminobutyric acid (GABA), noradrenaline (NE), dopamine (DA), acetylcholine (Ach), and serotonin (5-HT) - few reports have studied the effect of L-THE and PSE on these monoamines in the brain. Thus, this study also used the high performance liquid chromatography/mass spectroscopy (HPLC/ MS) method to the effect of these supplements on neurotransmitter levels in the brain.

We present the following article in accordance with the ARRIVE reporting checklist (available at http://dx.doi. org/10.21037/apm-21-663).

\section{Methods}

\section{Plant materials and reagents}

L-THE (20\%) was obtained from Novanat Co. Ltd. (Shanghai, China) and Neumentix PSE (rosmarinic acid 14.5-17.5\%, total phenolic 24-37\%) was purchased from Kemin Industries Inc. (Des Moines, IA, USA). Liquid chromatography mass spectroscopy (LC-MS)-grade acetonitrile and HPLC-grade methanol were purchased from Merck KGgA (Darmstadt, Germany). Formic acid (MS-grade) was purchased from CNW Technologies GmbH (Germany). Sodium pentobarbital, barbital, DA, Ach, NE, 5-HT, and GABA were supplied by SigmaAldrich (St. Louis, MO, USA). All other reagents were HPLC-grade and purchased from ThermoFisher Scientific (USA).

L-THE, PSE and L-THE/PSE mixtures were resuspended in distilled water, with the exception of sodium pentobarbital and barbital which were dissolved in physiological saline. For intragastric (i.g.) administration and intraperitoneal (i.p.) injection, all drugs and materials were prepared on the day of experiment. Control animals received the same volume of physiological saline and in the same route of administration.

\section{Animals}

A total of 252 male BALB/C mice (8 weeks old, 18-20 g) were obtained from SPF (Beijing) Biotechnology Co. Ltd. All mice were caged in specific-pathogen-free (SPF) animal housing with a temperature of $22 \pm 2{ }^{\circ} \mathrm{C}$, humidity of $55 \% \pm 5 \%$ and exposure to a 12 -hour light-dark cycle. Mice were fed with standard food and were able to drink freely for at least five days prior to commencing sleep testing. In this study, all experimental procedure and 
protocols were conducted in accordance with the American Physiological Society "Guiding principles in the Care and Use of Animals" and were approved by the Animal Ethics Committee of Animal Care Committee of the Fifth Medical Centre, Chinese People's Liberation Army General Hospital (ID: IACUC-2018-0022).

\section{Method for assessment of sleep improvement}

Male BALB/C mice were randomly divided into three groups for the pentobarbital-induced sleep test, subhypnotic pentobarbital-induced sleep test, and sodium barbital-induced sleep test, respectively. Each test contained 84 mice which were randomly allocated into 7 groups. In the mice experiments, L-THE, PSE, L-THE/PSE mixtures or physiological saline were administered intragastric (i.g.) for 30 continuous days. The assessment methods for measuring sleep improvement were carried out based on the Technical Standards for Testing \& Assessment of Health Food guidelines formulated by the Chinese Ministry of Health (22).

\section{Direct sleep test}

Mice were assessed as being awake or asleep 30 minutes after final oral administration of the supplement. This assessment was measured using loss of righting reflex; if the mouse was placed in the supine position and did not return to normal posture and fell asleep within 30-60 seconds, the normal reflex was considered to have disappeared and the mouse was assessed as being asleep (23).

\section{Pentobarbital-induced sleep test}

The sub-hypnotic pentobarbital (50 or $25 \mathrm{mg} / \mathrm{kg}$ ) was injected in each mouse 30 minutes after the last gavage to induce sleep. The number of mice asleep within 15 minutes was recorded and the total sleep time and sleep rate were calculated and recorded.

\section{Barbital sodium-induced sleep test}

To verify the synergistic effect of the plant materials and barbital sodium, a barbital sodium sleep incubation period test was conducted. The barbital sodium $(260 \mathrm{mg} / \mathrm{kg})$ was injected in each mouse 30 minutes after the last gavage to induce sleep. Sleep latency (the time between barbital sodium injection and sleep onset) was recorded. Mice that failed to fall asleep within 30 minutes were excluded from the experiment.

\section{Sample collection and preparation}

Two days after the final pentobarbital-induced sleep test, the mice were sacrificed by decapitation and their brains were rapidly dissected on an ice pad, then frozen and stored at $-80{ }^{\circ} \mathrm{C}$ until extraction.

At analysis, $100 \mathrm{mg}$ frozen brain tissue samples were weighed and homogenized in a 5 -fold volume of ice-cold methanol. The homogenates were centrifuged at 13,000 $\times \mathrm{g}$ for 20 minutes at $4{ }^{\circ} \mathrm{C}$, following which $50 \mu \mathrm{L}$ of the supernatant was transferred to a centrifuge tube which containing $150 \mu \mathrm{L}$ of methanol and acetonitrile $(1: 1, \mathrm{v} / \mathrm{v})$, which underwent 5 minutes of vortexing. Subsequently, these samples were centrifuged at 13,000 $\times \mathrm{g}$ for 10 minutes at $4{ }^{\circ} \mathrm{C}$. The $100 \mu \mathrm{L}$ of supernatants were collected and injected into the ultra-fast HPLC-MS system for analysis.

\section{LC-MS instrument and conditions}

The samples were analyzed using a Dionex Ultimate 3000 RS UPLC system connected to a Q-Exactive mass spectrometer (ThermoFisher Scientific, Germany). A Dionex Ultimate 3000 UHPLC system equipped with a WPS-3000 TRS autosampler, a TCC-3000 RS column oven and a HPG-3400 RS binary pump was used for UHPLC analysis. Thermo Hypersil Gold C18 column $(100 \mathrm{~mm} \times 2.1 \mathrm{~mm}, 3 \mu \mathrm{m})$ was employed for liquid chromatographic separation. The mobile phase consisted of $0.1 \%$ formic acid and $2.5 \mathrm{mM}$ ammonium formate in water (A) and acetonitrile (B) and the following gradient was employed: $0-1 \mathrm{~min}, 5-10 \% \mathrm{~B} ; 1-2.5 \mathrm{~min}, 10-95 \% \mathrm{~B} ; 2.5-$ $4.8 \mathrm{~min}, 95-5 \% \mathrm{~B}$. Flow rate was $0.3 \mathrm{~mL} / \mathrm{min}$ and injection volume was $5 \mu \mathrm{L}$.

Electrospray ionization (ESI) source was operated in positive mode. The ESI source parameters were: spray voltage $3.50 \mathrm{kV}$; capillary temperature $350^{\circ} \mathrm{C}$; sheath gas flow rate 40 units; auxiliary gas flow rate 10 units; sweep gas flow rate 0 units; $\mathrm{S}$ lens RF level 50 units and normalized collision energy 45 units. In full scan mode, the resolution was 70,000 with the lock mass function activated. Data was acquired and subsequently analyzed using Thermo Xcalibur software.

\section{Statistical analysis}

Testing dates were recorded and analyzed using Excel 
Table 1 Results of direct sleep test

\begin{tabular}{lcccc}
\hline Group & Dose & No. of animals & No. falling asleep & Proportion (\%) \\
\hline Control & 0 & 14 & 0 & 0 \\
L-THE & 82 & 14 & 0 & 0 \\
PSE & 184 & 14 & 0 & 0 \\
L-THE/PSE & $27 / 184$ & 14 & 0 & 0 \\
L-THE/PSE & $82 / 184$ & 14 & 0 & 0 \\
L-THE/PSE & $245 / 184$ & 14 & 0 & 0 \\
\hline
\end{tabular}

and SPSS 24.0, respectively, and the results are expressed as mean \pm standard error of the mean (SEM). Multiple comparisons data were analyzed using one-way analysis of variance (ANOVA) followed by the Student-Newman-Keuls test. For the test with a subthreshold dose of PENT the $\chi^{2}$ test or Fisher's exact probability test were used to compare the number of mice that fell asleep. $\mathrm{P}<0.05$ was considered statistically significant difference between groups.

\section{Results}

\section{Direct sleep test}

The sleep status of the mice administered L-THE, PSE and L-THE/PSE mixture were measured. The mice were observed 30 minutes after last oral gavage with the standard for sleep being loss of righting reflex. No mice with loss of righting reflex were observed. This result indicates that all L-THE, PSE and mixtures did not induce sleep directly in mice (Table 1).

\section{Effects of L-THE, PSE and mixture on sleep duration in the pentobarbital-induced $(50 \mathrm{mg} / \mathrm{kg})$ sleep model}

The sleep duration of the mice administered L-THE, PSE and L-THE/PSE mixture on pentobarbital-induced sleeping were measured. Compared with the control group, the L-THE $(82 \mathrm{mg} / \mathrm{kg}$ ) and mixture (L-THE $82 \mathrm{mg} / \mathrm{kg}$ and PSE $184 \mathrm{mg} / \mathrm{kg}$ ) groups significantly increased sleep duration in the mice $(\mathrm{P}<0.05)$. The group of PSE $(184 \mathrm{mg} / \mathrm{kg})$ prolonged the sleep duration in the mice, but the effect was not significant and the ratio was $15.65 \%$ (Figure 1A).

Subsequently, the dose of the PSE was kept unchanged and the induced changes in sleep duration at low, medium and high dose of L-THE (27, 82 and $245 \mathrm{mg} / \mathrm{kg}$ ) in the mixture on pentobarbital-induced sleeping were measured.
All three groups showed significantly prolonged sleep duration, but this effect reduced with increased dose of L-THE (Figure 1B).

Moreover, the mixtures at low and medium doses of L-THE showed a significantly increased sleep duration $(\mathrm{P}<0.05)$ compared with a single administration of PSE only, with ratios of $32.68 \%$ and $29.48 \%$, respectively. Compared with the group of L-THE only, the mixtures at low and medium doses showed a slight, non-significant increasing trend $(6.83 \%$ and $4.25 \%)$. Thus, the combined use of L-THE/PSE showed beneficial effects on sleep duration time.

Effects of L-THE and PSE on the number of mice that fell asleep in the sub-hypnotic pentobarbital-induced (25 mg/kg) sleep model

The number of mice that fell asleep within 30 minutes of L-THE, PSE and L-THE/PSE mixture administration in the sub-hypnotic pentobarbital-induced group was measured. Compared with the control group, the mixture (L-THE $27 \mathrm{mg} / \mathrm{kg}$ and PSE $184 \mathrm{mg} / \mathrm{kg}$ ) group significantly increased the number of the mice falling asleep $(\mathrm{P}<0.05)$. Other groups (except the L-THE/PSE mixtures of 245 and $184 \mathrm{mg} / \mathrm{kg}$, respectively) showed a positive effect of the supplement on the sub-hypnotic pentobarbital-induced sleep of animals, but the effect was not significant. Moreover, the number of the mice falling asleep in the mixture groups decreased with the increase dose of L-THE (Table 2).

\section{Effects of L-THE, PSE and mixture on sleep latency in the sodium barbital -induced (260 mg/kg) sleep model}

The sleep latency time of the mice administered L-THE, PSE and L-THE/PSE mixture on sodium barbital-induced sleeping were measured. Compared with the control and 

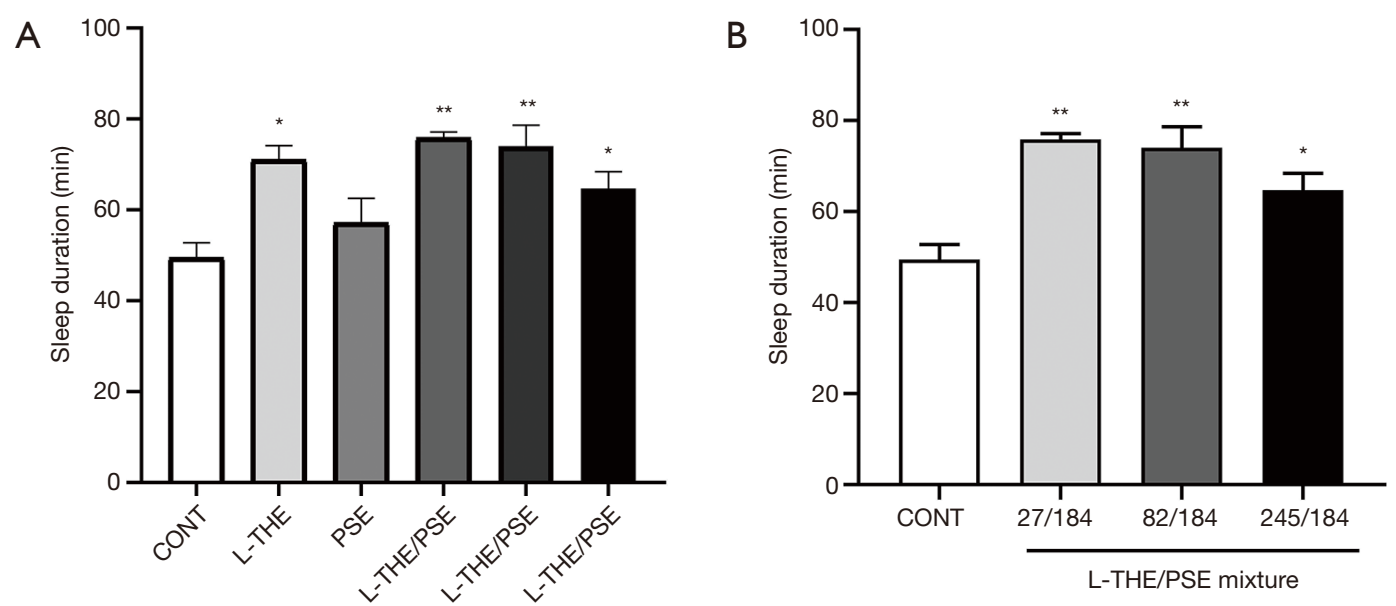

Figure 1 Pentobarbital-induced sleeping time of mice. Columns represent the mean \pm SEM. Symbols indicate significant differences compared to control group by Bonferroni test, as ${ }^{* *} \mathrm{P}<0.01,{ }^{*} \mathrm{P}<0.05$.

Table 2 Results of subthreshold dose of sub-hypnotic pentobarbital test

\begin{tabular}{lcccc}
\hline Group & Dose & No. of animals & No. falling asleep & Proportion (\%) \\
\hline Control & 0 & 14 & 3 & 21.42 \\
L-THE & 82 & 14 & 8 & 42.85 \\
PSE & 184 & 14 & 9 & 57.14 \\
L-THE/PSE & $27 / 184$ & 14 & 6 & $64.28^{\star}$ \\
L-THE/PSE & $82 / 184$ & 14 & 3 & 42.85 \\
L-THE/PSE & $245 / 184$ & 14 & 3 & 21.42 \\
\hline
\end{tabular}

${ }^{*} \mathrm{P}<0.05$, significantly different as compared to the control group.

PSE groups, the mixture group (L-THE $82 \mathrm{mg} / \mathrm{kg}$ and PSE $184 \mathrm{mg} / \mathrm{kg}$ ) showed significantly decreased sleep latency. The group given L-THE ( $82 \mathrm{mg} / \mathrm{kg}$ ) showed slightly shortened sleep latency, but the effect was not significant. Moreover, the combined use of a L-THE/PSE mixture also showed a not significant decrease in sleep latency compared with a single administration of L-THE (Figure $2 A$ ).

With respect to sleep latency, sleep time showed a tendency decrease in all doses of mixture groups. In particular, the mixture (L-THE $82 \mathrm{mg} / \mathrm{kg}$ and PSE $184 \mathrm{mg} / \mathrm{kg}$ ) group showed significantly reduced sleep latency (Figure $2 B$ ).

The mixtures at medium dose showed a significant decrease in sleep latency compared with a single administration of PSE, the ratio was $26.27 \%(\mathrm{P}<0.05)$. And compared with the L-THE only group, the mixtures at medium dose showed a slightly decreasing trend (14.51\%). Thus, the combined use of PSE/L-THE showed beneficial effects on sleep latency time.

\section{Levels of neurotransmitters in the brain}

The regulation of sleep and wakefulness are widely accepted to be associated with the levels of the corresponding neurotransmitters, such as GABA, NA, Ach, 5-HT and DA (Figure 3).

In the present study, we used the method of HPLC-MS to evaluate the sleep improvement efficacies of L-THE, PSE and a L-THE/PSE mixture by analyzing the changes in concentration of neurotransmitters in the brain in animal models. The levels of GABA and Ach in brain tissue were significantly increased compared with the group of control $(\mathrm{P}<0.05)$ (Figure 3). The group of L-THE $(82 \mathrm{mg} / \mathrm{kg})$ showed significantly reduced brain 5 -HT levels $(\mathrm{P}<0.05)$, and other groups showed decreased 5-HT concentration at 

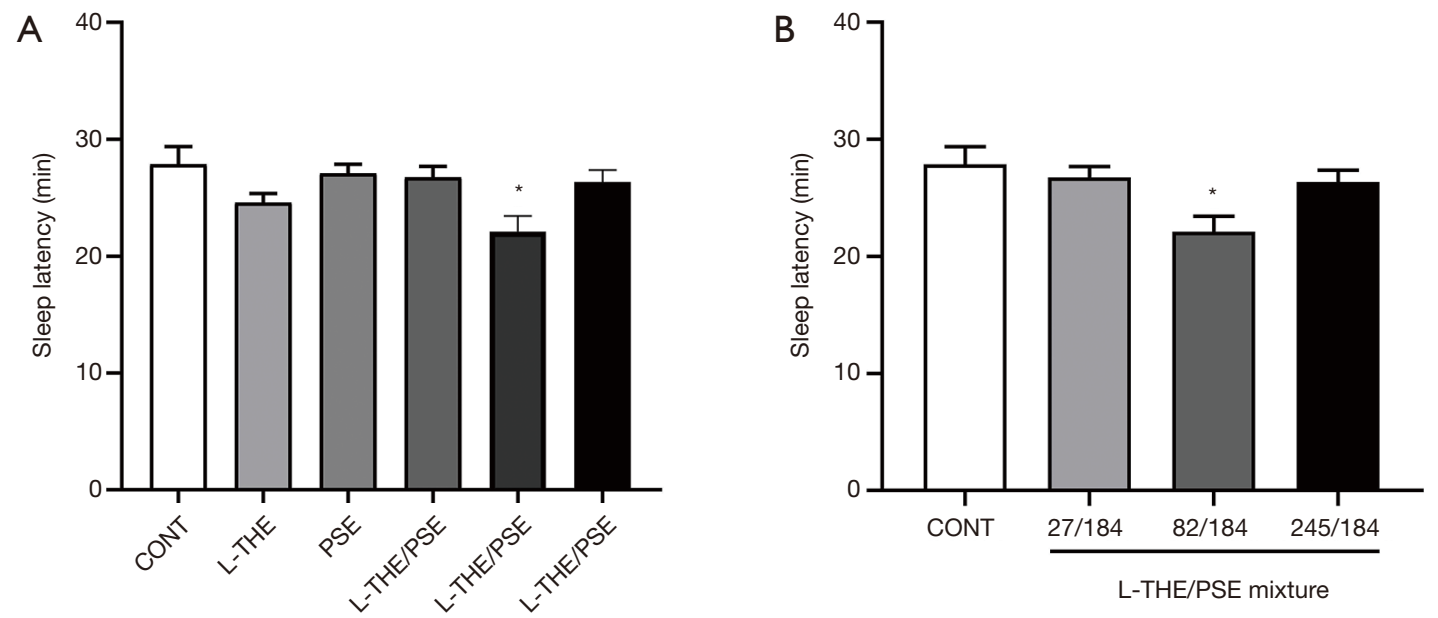

Figure 2 Sodium barbital-induced sleeping time of mice. Columns represent the mean \pm SEM. Symbols indicate significant differences compared to control group by Bonferroni test, as ${ }^{*} \mathrm{P}<0.05$.

a non-significant level compared with controls. The PSE $(184 \mathrm{mg} / \mathrm{kg})$ group showed a significant increase in brain DA level compared with other groups $(\mathrm{P}<0.05)$.

\section{Discussion}

Insufficient sleep increases the risk of various diseases and can lead to reduced quality of life and increased socioeconomic burden $(24,25)$. Recently, many studies have explored the effects of natural products on sleep in an effort to replace traditional hypnotic drugs which can have serious side effects. L-THE is a known agent for treating sleep disorders and has also been used in various other settings to induce sedation and relaxation (14). The sleep improvement properties of PSE have also been studied previously in an older demographic (aged 50 to 70 years), and one of its ingredients, rosmarinic acid, has been shown to improve NREM sleep and $\delta$-wave activity in the brain $(21,26)$.

It is well known that drugs with sedative-hypnotic properties can prolong the sleep time caused by barbiturates. Our tests, including the pentobarbital-induced sleeping time test, subthreshold dose of sodium pentobarbital test, and barbital sodium sleep incubation period tests showed that the L-THE and mixtures had significant effects of sleep improvement. In this study, we observed that mixtures in different doses not only prolonged sleep duration and increased the number of the mice falling asleep in the pentobarbital-induced sleep model, but also reduce sleep latency in the sodium barbital-induced sleep model.

Pentobarbital sodium and barbital sodium are known
$\mathrm{GABA}_{\mathrm{A}}$ receptor agonists. The activation of $\mathrm{GABA}_{\mathrm{A}}$ receptors shows sedative, hypnotic and anticonvulsive effects (27). When GABA or $\mathrm{GABA}_{\mathrm{A}}$ receptor agonists bind to $\mathrm{GABA}_{\mathrm{A}}$ receptors, $\mathrm{Cl}^{-}$ion channels open and these ions enter the cells, resulting in cell hyperpolarization, reduced cell excitability and promotion of sleep. Rosmarinic acid is one of the main components of PSE. Rosmarinic acid exhibits activation of the $\mathrm{GABA}_{\mathrm{A}}$ energy system by increasing $\mathrm{Cl}^{-}$ion influx, thereby enhancing pentobarbitalinduced sleep behavior and non-rapid eye movement (NREM) sleep (21). L-THE has been reported to inhibit caffeine-induced convulsions and increase GABA levels in the brains of mice (28). This indicates that the hypnotic effects of L-THE and mixtures may be due to interaction with GABAergic systems.

Using the methods from The Chinese Ministry of Heath for improving sleep function adopted in this study, our results showed that the group of mixtures (27/184 and $82 / 184 \mathrm{mg} / \mathrm{kg}$ ) had a sleep-promoting effect and certain sedative effects, showing synergistic action of L-THE/ PSE mixture on sleep behaviors compared with control and mono-therapy L-THE and PSE groups. Furthermore, the sleep-promoting effect of the mixture group may be regulated by neurotransmitter systems, such as GABA, Ach and 5-HT.

The sleep-wake cycle is regulated by excitatory and inhibitory neuronal systems. Many neurotransmitters, such as GABA and Ach, are involved in sleep regulation (29). GABA is reported as a major inhibitory neurotransmitter in the central nervous system that not only mediates fast 
A

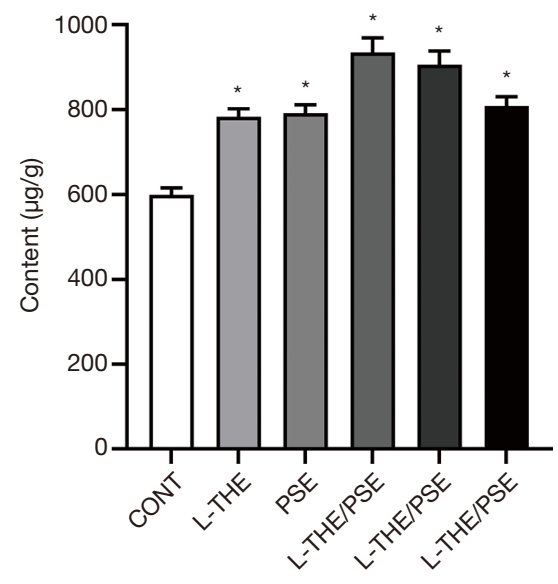

GABA

D

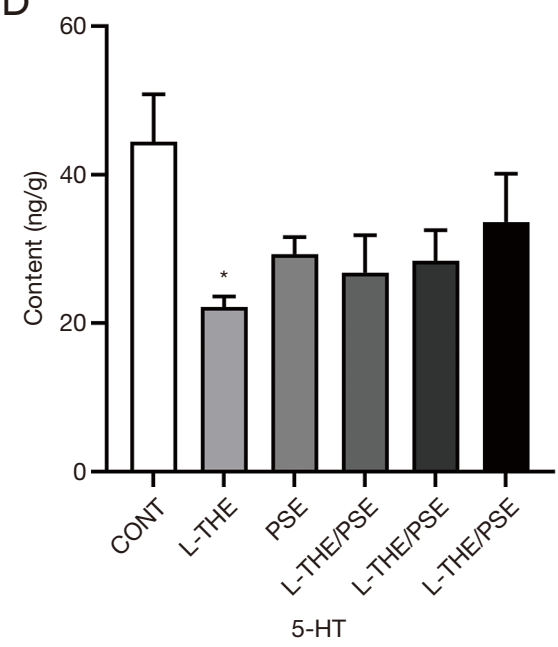

B

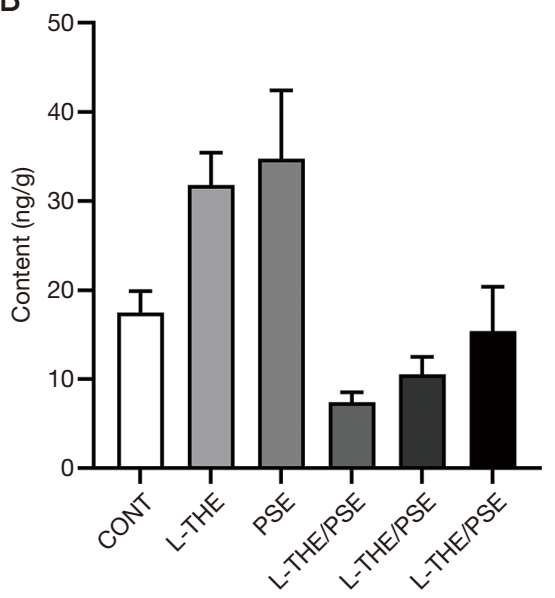

NE

$\mathrm{E}$

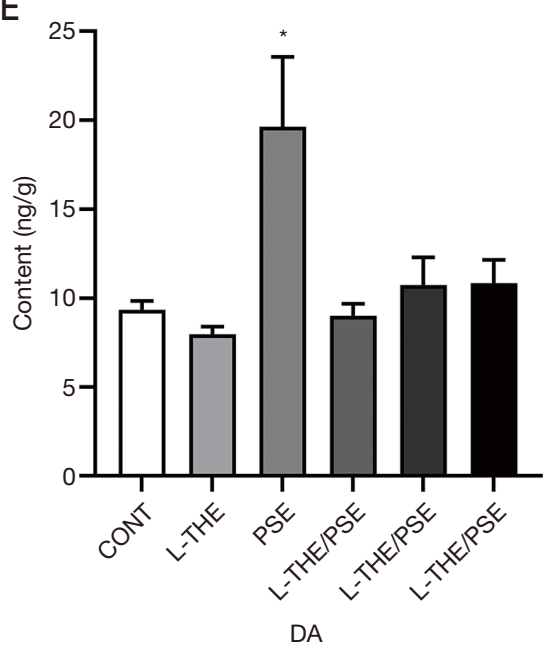

C

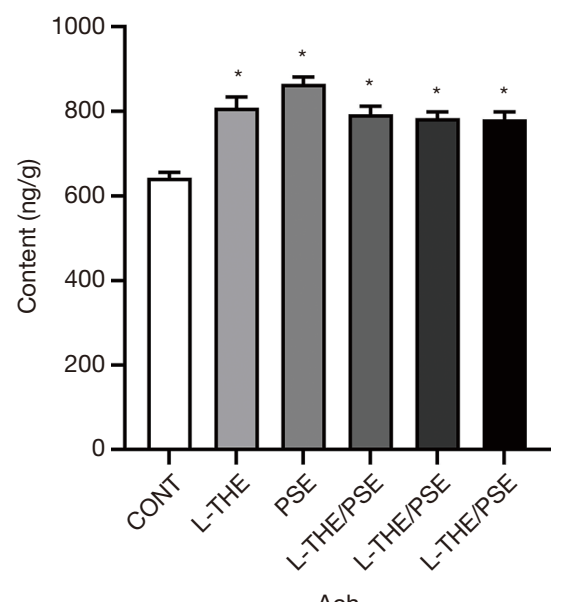

Ach

Figure 3 The levels of the five differential neurotransmitters. (A) The content of GABA in the brain tissue of mice after intervention with different products. (B) The content of NE in the brain tissue of mice after intervention with different products. (C) The content of Ach in the brain tissue of mice after intervention with different products. (D) The content of 5-HT in the brain tissue of mice after intervention with different products. (E) The content of DA in the brain tissue of mice after intervention with different products. Columns represent the mean $\pm \operatorname{SEM}(\mathrm{n}=8)$. Symbols indicate significant differences compared to control group by Bonferroni test, as ${ }^{*} \mathrm{P}<0.05$.

synaptic inhibition via $\mathrm{GABA}_{\mathrm{A}}$ receptors, but also regulates the excitability of distinct neuronal networks via extrasynaptic $\mathrm{GABA}_{\mathrm{A}}$ receptors (30). Therefore, the GABA system plays an important role in anxiety and insomnia. Our results showed that all model groups produced a significant increase in GABA concentration levels in the brain compared with the control group. It has also been reported that Ach plays a role in rapid eye movement (REM) sleep (31), with one study showing that increased release of Ach in the pontine reticular formation promoted REM sleep in mice (32). Meanwhile, NA often acts as a wake- promoting neurotransmitter (33); the balance between Ach and NA in the brain may therefore play an important role in the regulation of REM sleep (34). Previous research has shown that sole administration of Ach inhibitors promoted wakefulness, but Ach inhibitors administered after a decrease in NA levels promoted REM sleep (35).

In our study, a significant increase in Ach and decrease in NA levels in the brain were observed in all model groups. Furthermore, the level of 5-HT also significantly decreased compared with the control group and appeared to act as a wake-promoting neurotransmitter. Based on our data, it 
can be inferred that a L-THE/PSE mixture may inhibit NA and 5-HT neurotransmitter activity and reduce their levels in the brain. Furthermore, the results suggest that L-THE/ PSE mixtures may also activate the GABAergic neurons and increase the levels of GABA in the brain, consequently promoting sleep.

Our study suggests that the combined use of L-THE and PSE to improve sleep is more effective than the use of L-THE or PSE alone. The superior effect of the L-THE and PSE mixture is likely to be mediated by neurotransmitter systems, including the GABAergic system in the brain. Our results suggest that a L-THE and PSE mixture could serve as a plant material alternative for treating insomnia and sleep disorders, and there is potential for new foods to be developed to treat these conditions using a mixture of these ingredients.

\section{Acknowledgments}

Funding: None.

\section{Footnote}

Reporting Checklist: The authors have completed the ARRIVE reporting checklist. Available at http://dx.doi. org/10.21037/apm-21-663

Data Sharing Statement: Available at http://dx.doi. org/10.21037/apm-21-663

Conflicts of Interest: All authors have completed the ICMJE uniform disclosure form (available at http://dx.doi. org/10.21037/apm-21-663). Dr. Jia is affiliated to Inner Mongolia Yili Industrial Group Co., Ltd. Dr. Chen is affiliated to Bonnysci International Science \& Technology Co., Ltd. Dr. Zhao is affiliated to Inner Mongolia Dairy Technology Research Institute Co., Ltd. The other authors have no conflicts of interest to declare.

Ethical Statement: The authors are accountable for all aspects of the work and ensuring that questions related to the accuracy or integrity of any part of the work are appropriately investigated and resolved. All experimental procedure and protocols were conducted in accordance with the American Physiological Society "Guiding principles in the Care and Use of Animals" and were approved by the Animal Ethics Committee of Animal Care Committee of The Fifth Medical Centre, Chinese
PLA People's Liberation Army General Hospital (ID: IACUC-2018-0022).

Open Access Statement: This is an Open Access article distributed in accordance with the Creative Commons Attribution-NonCommercial-NoDerivs 4.0 International License (CC BY-NC-ND 4.0), which permits the noncommercial replication and distribution of the article with the strict proviso that no changes or edits are made and the original work is properly cited (including links to both the formal publication through the relevant DOI and the license). See: https://creativecommons.org/licenses/by-ncnd/4.0/.

\section{References}

1. Xie Z, Chen F, Li WA, et al. A review of sleep disorders and melatonin. Neurol Res 2017;39:559-65.

2. Doghramji K. The epidemiology and diagnosis of insomnia. Am J Manag Care 2006;12:S214-20.

3. Liu WL, Wu BF, Shang JH, et al. Moringa oleifera Lam Seed Oil Augments Pentobarbital-Induced Sleeping Behaviors in Mice via GABAergic Systems. J Agric Food Chem 2020;68:3149-62.

4. Sánchez-Cárdenas AG, Navarro-Gerrard C, NellenHummel $\mathrm{H}$, et al. Insomnia. A severe health care problem. Rev Med Inst Mex Seguro Soc 2016;54:760-9.

5. Matheson E, Hainer BL. Insomnia: Pharmacologic Therapy. Am Fam Physician 2017;96:29-35.

6. Graham HN. Green tea composition, consumption, and polyphenol chemistry. Prev Med 1992;21:334-50.

7. Kakuda T. Neuroprotective effects of theanine and its preventive effects on cognitive dysfunction. Pharmacol Res 2011;64:162-8.

8. Cicero AFG, Fogacci F, Banach M. Botanicals and phytochemicals active on cognitive decline: The clinical evidence. Pharmacol Res 2018;130:204-12.

9. Deb S, Dutta A, Phukan BC, et al. Neuroprotective attributes of L-theanine, a bioactive amino acid of tea, and its potential role in Parkinson's disease therapeutics. Neurochem Int 2019;129:104478.

10. Li C, Yan Q, Tang S, et al. L-Theanine Protects H9C2 Cells from Hydrogen Peroxide-Induced Apoptosis by Enhancing Antioxidant Capability. Med Sci Monit 2018;24:2109-18.

11. Lopes Sakamoto F, Metzker Pereira Ribeiro R, Amador Bueno A, et al. Psychotropic effects of L-theanine and its 
clinical properties: From the management of anxiety and stress to a potential use in schizophrenia. Pharmacol Res 2019;147:104395.

12. Jang HS, Jung JY, Jang IS, et al. L-theanine partially counteracts caffeine-induced sleep disturbances in rats. Pharmacol Biochem Behav 2012;101:217-21.

13. Lyon MR, Kapoor MP, Juneja LR. The effects of L-theanine (Suntheanine ${ }^{\circledR}$ ) on objective sleep quality in boys with attention deficit hyperactivity disorder (ADHD): a randomized, double-blind, placebocontrolled clinical trial. Altern Med Rev 2011;16:348-54.

14. Kim S, Jo K, Hong KB, et al. GABA and l-theanine mixture decreases sleep latency and improves NREM sleep. Pharm Biol 2019;57:65-73.

15. Bian Y, Yamashita T, Taira Y, et al. A Polyphenolic Complex Attenuates Inflammatory Response and Blood- Brain Barrier Disruption. Curr Neurovasc Res 2020;17:286-93.

16. Farr SA, Niehoff ML, Ceddia MA, et al. Effect of botanical extracts containing carnosic acid or rosmarinic acid on learning and memory in SAMP8 mice. Physiol Behav 2016;165:328-38.

17. Taira Y, Yamashita T, Bian Y, et al. Antioxidative effects of a novel dietary supplement Neumentix in a mouse stroke model. J Stroke Cerebrovasc Dis 2020;29:104818.

18. Falcone PH, Nieman KM, Tribby AC, et al. The attention-enhancing effects of spearmint extract supplementation in healthy men and women: a randomized, double-blind, placebo-controlled, parallel trial. Nutr Res 2019;64:24-38.

19. Nunes S, Madureira AR, Campos D, et al. Therapeutic and nutraceutical potential of rosmarinic acid-Cytoprotective properties and pharmacokinetic profile. Crit Rev Food Sci Nutr 2017;57:1799-806.

20. Lee YW, Kim DH, Jeon SJ, et al. Neuroprotective effects of salvianolic acid $\mathrm{B}$ on an $\mathrm{A} \beta 25-35$ peptide-induced mouse model of Alzheimer's disease. Eur J Pharmacol 2013;704:70-7.

21. Kwon YO, Jin Tae Hong JT, Ki-Wan Oh KW, et al. Rosmarinic Acid Potentiates Pentobarbital-Induced Sleep Behaviors and Non-Rapid Eye Movement (NREM) Sleep through the Activation of GABA A-ergic Systems. Biomol Ther (Seoul) 2017;25:105-11.

22. Zhao W, Li Y, Ma W, et al. A study on quality components and sleep-promoting effects of GABA black tea. Food Funct 2015;6:3393-8.

23. Deng L, Shi AM, Wang Q. Sedative-hypnotic and anxiolytic effects and the mechanism of action of aqueous extracts of peanut stems and leaves in mice. J Sci Food Agric 2018;98:4885-94.

24. Yuen KM, Pelayo R. Socioeconomic Impact of Pediatric Sleep Disorders. Sleep Med Clin 2017;12:23-30.

25. Wickwire EM, Shaya FT, Scharf SM, et al. Health economics of insomnia treatments: The return on investment for a good night's sleep. Sleep Med Rev 2016;30:72-82.

26. Herrlinger KA, Nieman KM, Sanoshy KD, et al. Spearmint Extract Improves Working Memory in Men and Women with Age-Associated Memory Impairment. J Altern Complement Med 2018;24:37-47.

27. Choi JJ, Oh EH, Lee MK, et al. Gastrodiae Rhizoma Ethanol Extract Enhances Pentobarbital-Induced Sleeping Behaviors and Rapid Eye Movement Sleep via the Activation of GABA A -ergic Transmission in Rodents. Evid Based Complement Alternat Med 2014;2014:426843.

28. Kimura R, Murata T. Influence of alkylamides of glutamic acid and related compounds on the central nervous system. I. Central depressant effect of theanine. Chem Pharm Bull (Tokyo) 1971;19:1257-61.

29. Yan Y, Li Q, Du HZ, et al. Determination of five neurotransmitters in the rat brain for the study of the hypnotic effects of Ziziphi Spinosae Semen aqueous extract on insomnia rat model by UPLC-MS/MS. Chin J Nat Med 2019;17:551-60.

30. Yan MZ, Chang Q, Zhong Y, et al. Lotus Leaf Alkaloid Extract Displays Sedative-Hypnotic and Anxiolytic Effects through GABAA Receptor. J Agric Food Chem 2015;63:9277-85.

31. Kodama T, Takahashi Y, Honda Y. Enhancement of acetylcholine release during paradoxical sleep in the dorsal tegmental field of the cat brain stem. Neurosci Lett 1990;114:277-82.

32. He B, Bi K, Jia Y, et al. Rapid analysis of neurotransmitters in rat brain using ultra-fast liquid chromatography and tandem mass spectrometry: application to a comparative study in normal and insomnic rats. J Mass Spectrom 2013;48:969-78.

33. Schwartz MD, Kilduff TS. The Neurobiology of Sleep and Wakefulness. Psychiatr Clin North Am 
2015;38:615-44.

34. Jones BE. From waking to sleeping: neuronal and chemical substrates. Trends Pharmacol Sci 2005;26:578-86.

35. Cao Q, Jiang Y, Cui SY, et al. Tenuifolin, a saponin derived

Cite this article as: Zhang $\mathrm{Y}$, Jia $\mathrm{X}$, Chen $\mathrm{X}$, Liu Y, Zhao Z, Hao J, Wu R, Feng H, Ren X. L-theanine and Neumentix mixture improves sleep quality and modulates brain neurotransmitter levels in mice. Ann Palliat Med 2021;10(4):4572-4581. doi: 10.21037/apm-21-663 from Radix Polygalae, exhibits sleep-enhancing effects in mice. Phytomedicine 2016;23:1797-805.

(English Language Editor: M. Hawkins) 